\title{
New contrast agents and technologies for myocardial contrast echocardiography
}

\author{
Daniele Rovai, Mark J Monaghan
}

Myocardial contrast echocardiography is a new method for evaluating myocardial perfusion. However, it relies on the marriage of two evolving technologies; ultrasound contrast agents and ultrasound imaging instrumentation. To date, these technologies have only been available to a limited number of researchers throughout the world. Within the next 12 months, both agents and specialised ultrasound instruments will be commercially available, making this new methodology open to widespread clinical application. Moreover, echocardiographic evaluation of myocardial perfusion should have a major role in the management of both chronic and acute ischaemic situations, myocardial infarction, and assessment of myocardial viability. This review examines the current and likely future status of both contrast agents and machines in this context. In addition, the probable clinical role of the methodology, as a potential replacement for nuclear techniques, is discussed

In patients with coronary artery disease the study of myocardial perfusion is of great clinical relevance and is currently investigated by nuclear medicine techniques. Nuclear cardiology, however, is limited by contamination risks, by a limited availability of the equipment, and by considerable expense. Furthermore, the current role of nuclear medicine in cardiology is mainly confined to stable patients, while acutely ill patients rarely benefit from the information regarding myocardial perfusion.

An alternative method of studying myocardial perfusion is echocardiography following the injection of ultrasound contrast agents directly into the coronary circulation. ${ }^{12}$ This invasive approach, however, is limited to applications within the cardiac catheterisation laboratory. To overcome this limitation, investigators, pharmaceutical companies, and ultrasound industries have sought to develop a method that could achieve myocardial enhancement following peripheral venous injection of ultrasound contrast agents.

Wider use of this methodology might have an important impact on clinical cardiology, as myocardial perfusion could be evaluated noninvasively, requiring only the use of widely available ultrasound scanners that have been suitably adapted. Additionally, the study of myocardial perfusion by contrast echocardiography would make information on perfusion available to a large population of patients in different settings, from the emergency room to the outpatient clinic. This non-invasive approach to myocardial contrast echocardio- graphy stands on three main pillars: new ultrasound contrast agents, new equipment, and clinical models for myocardial perfusion studies.

\section{New contrast agents}

Left ventricular cavity opacification can be obtained in the majority of patients after venous injection of lung-crossing agents such as Albunex (MBI, USA) ${ }^{3}$ and Levovist (Schering AG, Germany). ${ }^{4}$ However, neither of these agents is able to opacify the myocardium consistently using conventional signal processing and imaging modalities. In an attempt to visualise myocardial perfusion by intravenous Albunex injection, Villaneuva et $a l^{5}$ injected dogs with a dose of this agent 10 times that previously used for diagnostic purposes. They selected microbubbles with a diameter larger than those used for conventional imaging and injected the contrast agent directly into the right atrial cavity. In addition, the animals were premedicated with dipyridamole (to increase the ratio between coronary blood flow and cardiac output) and, finally, the echocardiographic images were digitally subtracted and colour coded. Although this approach allowed myocardial perfusion to be visualised in experimental animals, it cannot be used in the clinical setting.

Recently, a new generation of ultrasound contrast agents has been produced characterised by a prolonged persistence in the circulation. This persistence is related to the shell stability and the gases contained in the microbubbles, which have a higher density than air and a low solubility in blood. As shown in fig 1 , the echo contrast effect generated by an agent spontaneously declines over time. This spontaneous decay in contrast intensity resembles that of radioisotopes and is mainly caused by the diffusion of the gases from the microbubbles in the surrounding medium. Thus, the microbubbles containing gases with low solubility in blood are characterised by a prolonged persistence and in vivo stability.

A precursor of long persistence contrast agents is the agent EchoGen (Sonus Pharmaceutical, USA), which is based on dodecafluoropentane. This gas, in addition to the high density and low solubility in blood, displays an interesting property, a phase shift at $28.5^{\circ} \mathrm{C}$. Thus, the agent is a fluid at room temperature and shifts to a gaseous phase at body temperature. Both cardiac cavities and the myocardium show contrast enhancement 


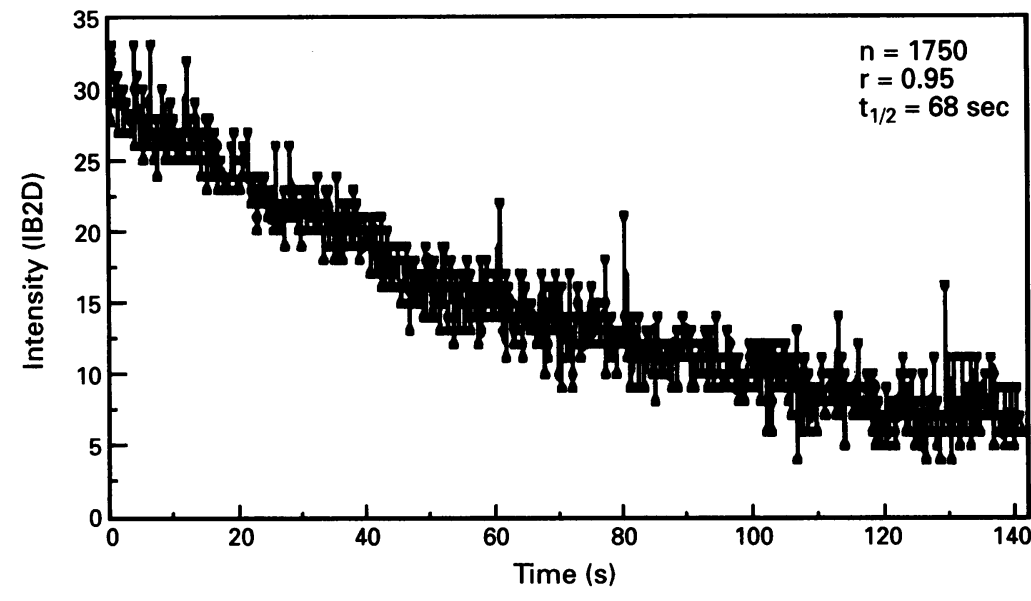

Figure 1 Spontaneous decay in echo contrast intensity. In this experiment the contrast agent SHU 454 (Echovist) was injected in a recirculating flow model. After the equilibrium was reached, signal intensity declined because of the diffusion of gases from the microbubbles in the surrounding medium. The decay half time of Echovist in saline corresponded to 68 seconds. IB2D, 3D integrated backscatter index.

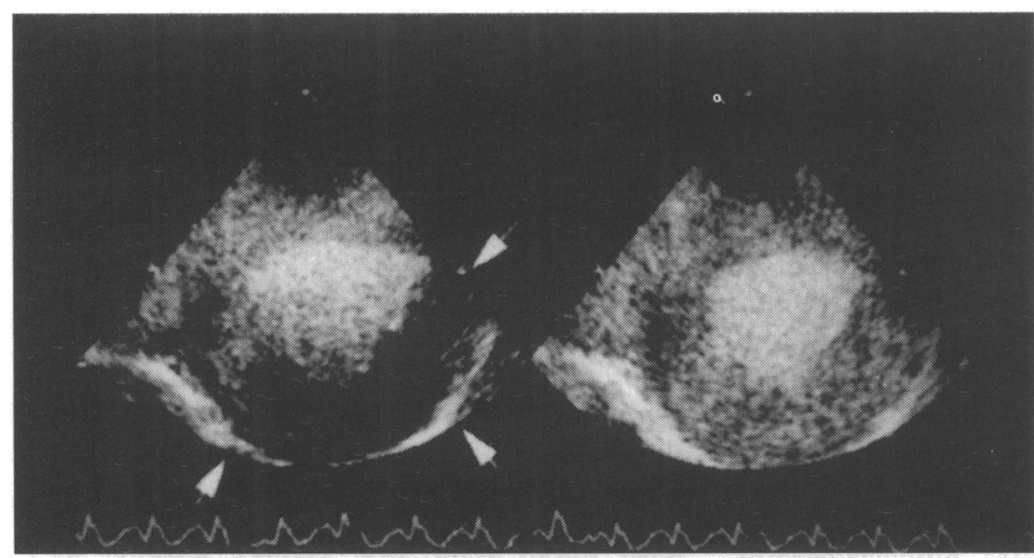

Figure 2 The myocardium perfused by the left anterior descending and the right coronary artery shows a contrast enhancement one minute after intravenous administration of the echo contrast agent BR1 (left panel). The myocardium perfused by the left circumflex coronary artery show a contrast effect three minutes later (right panel).

soon after venous administration. Later on, the contrast is washed out from the cardiac cavities whereas the myocardium maintains contrast enhancement, which can persist for several minutes. Recently, Grayburn et $a l^{6}$ administered EchoGen intravenously in a canine model of acute coronary occlusion. Myocardial contrast echocardiography accurately defined the extent of the area of myocardium at risk and the infarct size. The main limitation of this agent is its potential toxicity; pulmonary artery pressure and pulmonary vascular resistance increase at high doses of contrast, while arterial oxygen satura- tion and cardiac output decrease. A new formulation of this agent that allows a dense myocardial opacification without negative haemodynamic effects is under investigation. ${ }^{7}$

Other new generation contrast agents are also characterised by an elevated in vivo stability but show the ability freely to cross the microcirculation in the absence of any tissue deposit. Compared with Albunex and Levovist, the behaviour of these agents is closer to that of intravascular free flowing tracers. These agents include perfluorocarbon exposed sonicated dextrose albumin, ${ }^{8}$ FS069 (MBI), ${ }^{9}$ BR1 (Bracco Research, Switzerland), ${ }^{10}$ Imagent(r)US (Alliance Pharmaceutical Corp, USA), ${ }^{11}$ and Aerosomes. ${ }^{12}$ There are several others under investigation. The table illustrates many of the ultrasound contrast agents that are currently under development or available.

The intravenous injection of these agents results in an enhancement of the ultrasound signal in the ventricular cavities, which can last up to several minutes. During peak contrast enhancement of the left ventricular cavity, myocardial opacification has been observed with all agents. Myocardial contrast echo effect obtained by intravenous contrast echocardiography is expected to reflect the presence of microbubbles in the coronary circulation and thus myocardial perfusion. However, contrast enhancement of the myocardium could also be ascribed to a blooming effect induced by the huge number of microbubbles in the left ventricular cavity. Figure 2 shows that myocardial contrast effect does reflect true myocardial perfusion. In this dog, the left circumflex coronary artery was cannulated and perfused via a bypass with blood taken from the femoral artery (fig 3); a reservoir filled with blood was present in series in the bypassing circuit and delayed the arrival of blood in the circumflex perfusion territory. After intravenous contrast administration, the myocardium perfused by the left anterior descending and the right coronary artery early showed contrast enhancement (fig 2, left panel), while the myocardium perfused by the circumflex coronary artery showed a late contrast effect (fig 2, right panel). Furthermore, several experiments have been carried out in different laboratories that support the concept that myocardial echo enhancement after venous administration of the most recent generation of contrast agents reflects myocardial perfusion and may permit quantitative assessment of perfusion deficits.

Ultrasound contrast agents available or under development

\begin{tabular}{llll}
\hline Agent & Manufacturer & Type & Encapsulated Gas \\
\hline Albunex/Infoson & Mallinckrodt (USA) & Albumin microsphere & Air \\
NC100,100 & Nycomed (Norway) & Confidential & Confidential \\
Levovist & Schering (Germany) & Galactose palmitic acid & Air \\
Aerosomes/MRX115 & ImaRx (USA) & Lipid microbubble & Perfluoropropane \\
Quantison (Depot) & Andaris (UK) & Albumin microsphere & Air \\
Echogen & Sonus (USA) & Phase-shift & Dodecofluoropentane \\
BY 963 & Byk-Gulden (Germany) & Phospholipid & Air \\
FS069 & MBI (USA) & Albumin microsphere & Pentafluoropentane \\
BR1/Sonovue & Bracco (Switzerland) & Lyophilisate & SF6 \\
Imagent/AFO150 & Alliance (USA) & Buffered surfactant & Perfluorohexane \\
Sonovist/SHU 563A & Schering & Cyanocrylate particle & Air \\
Echovist & Schering & Galactose & Air \\
\hline
\end{tabular}




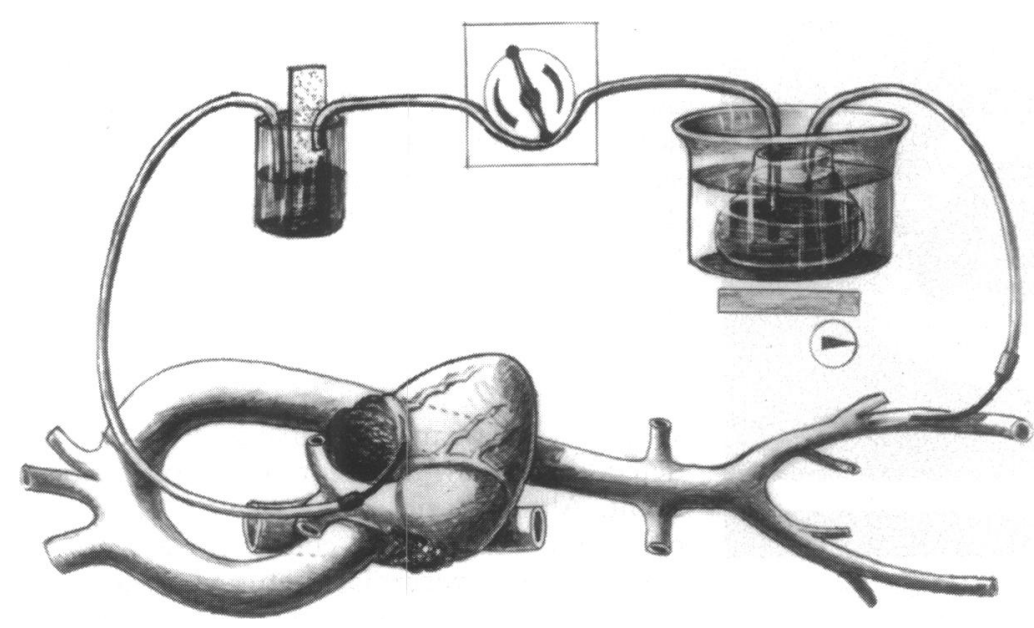

Figure 3 Experimental animal model. The left circumflex coronary artery is perfused via a bypass with blood taken from the femoral artery; a reservoir is present in series in the bypassing circuit.

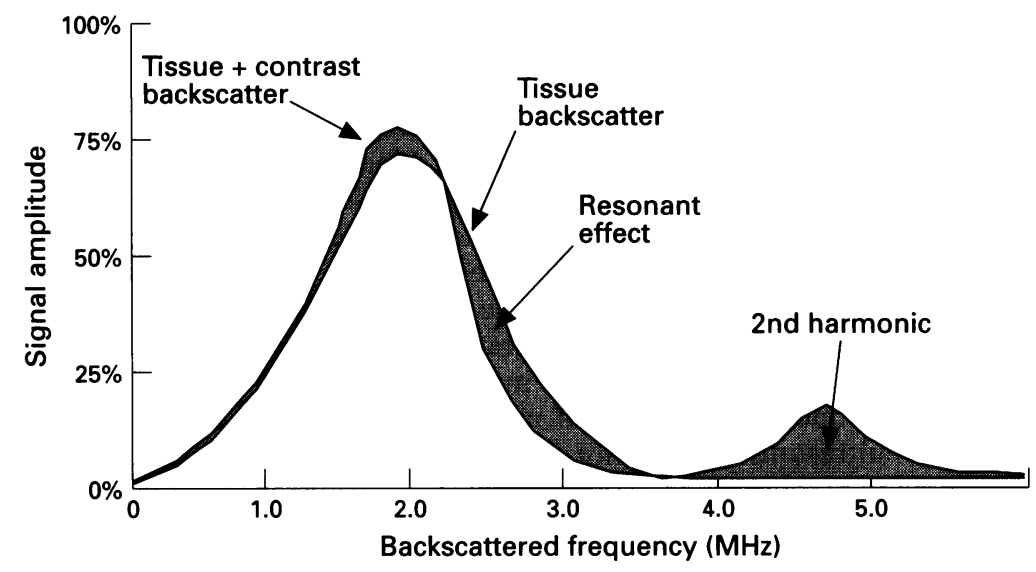

Figure 4 Diagrammatic representation of the principle behind 2nd harmonic contrast imaging. A graph of backscattered ultrasound signal amplitude against frequency is illustrated for tissue alone and tissue plus contrast. The tissue signal is centred around the transmitted fundamental frequency of $2 \mathrm{MHz}$. However, when a contrast agent is introduced into the tissues, it causes a small increment in signal amplitude at the fundamental (used in conventional imaging), an absorption of energy around the resonant frequency $(2 \cdot 5 \mathrm{MHz})$, and an increase in signal at the 2 nd harmonic. The latter two effects may be detected by frequency specific imaging technology and allow better contrast for tissue discrimination than conventional imaging methods.

\section{New technologies}

Despite the development of new contrast agents, which have improved stability and in vivo persistence, it has been difficult to demonstrate reliably myocardial perfusion using conventional echocardiographic techniques. Therefore, attempts have been made to explore other imaging technologies, which may offer improved sensitivity for detection of the small quantities of contrast agent within the myocardium.

Many of these new technologies have utilised the fact that some ultrasound contrast microspheres have resonant properties. ${ }^{1314}$ This means that, when exposed to an ultrasound field where the resonance frequency of the contrast microspheres lies within the bandwidth of transmitted ultrasound, the characteristics of the backscattered signal will be altered in some way. Contrast microspheres absorb energy at their resonant frequency and that absorbed energy is re-emitted at harmonics of the resonant frequency ${ }^{15}$ (fig 4). While con- trast agents do cause an increase in backscattered ultrasound intensity at the fundamental frequency (centre frequency of the transducer), the increment in intensity over the signal reflected from myocardial tissue is relatively small. This can make it difficult to evaluate the presence of the agent within the tissue. However, by examining specifically the signal content at the resonant frequency or its harmonic (especially the 2nd harmonic), there is much greater difference in signal level between tissue alone and tissue plus contrast. This makes detection of the presence of the contrast agent and its distribution much easier to appreciate.

While the tissue signal at the 2 nd harmonic, which is achieved by transmitting at one frequency and then selectively receiving at double that frequency, is relatively low, the contrast agent generates a high amplitude signal, which is easier to detect. This technology is relatively easy to implement, either by modification to transducer design or by using broadband width transducers and digital input filtering. It has been shown to demonstrate myocardial perfusion as well as perfusion in other organs such as liver and kidney following low volume intravenous injections. ${ }^{15}$ It is unlikely that 2 nd harmonic imaging is the final stage in the development of contrast agent imaging technology.

Recent studies have indicated that exposure of contrast microbubbles to diagnostic ultrasound fields results in their destruction. This clearly limits the potential effectiveness of ultrasound detection of perfusion. Methods to limit this phenomenon have been investigated including reducing the transducer output power, thereby decreasing spatial ultrasound intensity. Reductions in temporal ultrasound exposure may also be achieved by reconfiguring the echocardiography system so that intermittent rather than continuous imaging is performed. ${ }^{816}$ This means that only one frame of an echocardiographic image is generated every cardiac cycle (or every other cardiac cycle) rather than the normal imaging mode when approximately 30 frames per second are generated. By employing intermittent imaging, significant improvements in detection of myocardial perfusion have been demonstrated, ${ }^{17}$ as destruction of contrast microspheres is considerably reduced and microspheres that are destroyed are usually replenished before the next frame is created. One limitation of this technique is that a real time image is no longer displayed, which may have limitations for temporal analysis and visual appreciation of contrast appearance and disappearance. Improvements in image processing and display technology are likely to be employed to overcome these limitations.

However, it is also possible that the phenomenon of contrast microbubble destruction could be turned to advantage in the future, as contrast microbubbles could be used for drug delivery, using localised higher intensity ultrasound to destroy the microspheres and release a drug at desired sites. In addition, it may be possible to adjust ultrasound output power 


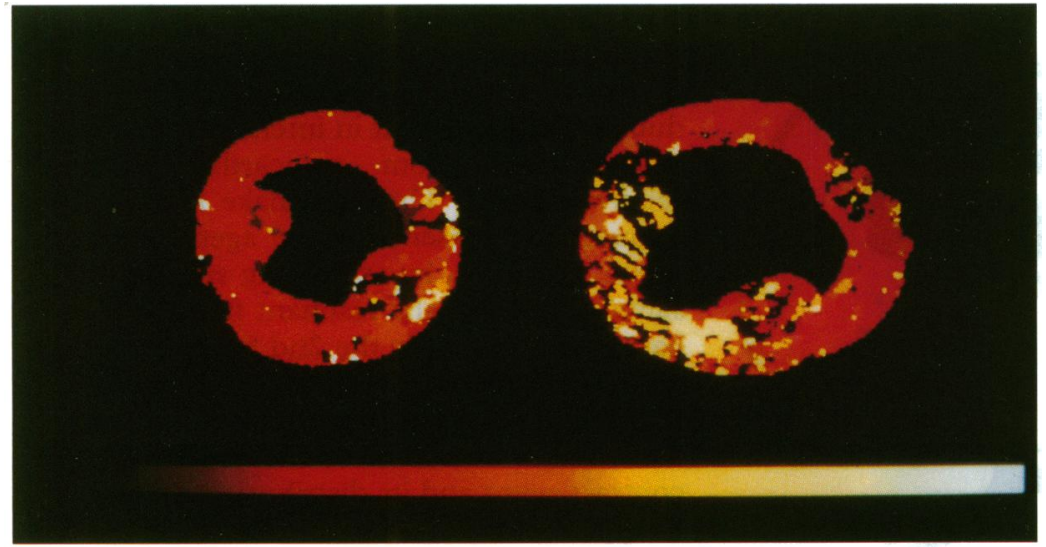

Figure 5 Colour coded functional images of myocardial perfusion. The time from contrast injection into the aortic root up to myocardial contrast appearance is displayed in a colour scale (left, baseline; right, ischaemia). In the presence of a severe stenosis of the left circumflex coronary artery myocardial contrast appearance is delayed and the ischaemic myocardium shows up as yellow.

levels, so that contrast microspheres are destroyed at exactly the same rate that they are replenished, within a given myocardial bed. Myocardial perfusion, which was occurring at a rate higher than the destruction rate, would result in a gradual increase in segment intensity, whereas lower levels of perfusion would mean that more microbubbles were being destroyed than being replenished and so intensity would decrease. In theory, this would allow this phenomenon to be used for measuring myocardial perfusion.

These technologies are still very much in their infancy, however, many ultrasound instrument manufacturers are introducing harmonic imaging as a facility on their systems. In addition, quantitative software is now available

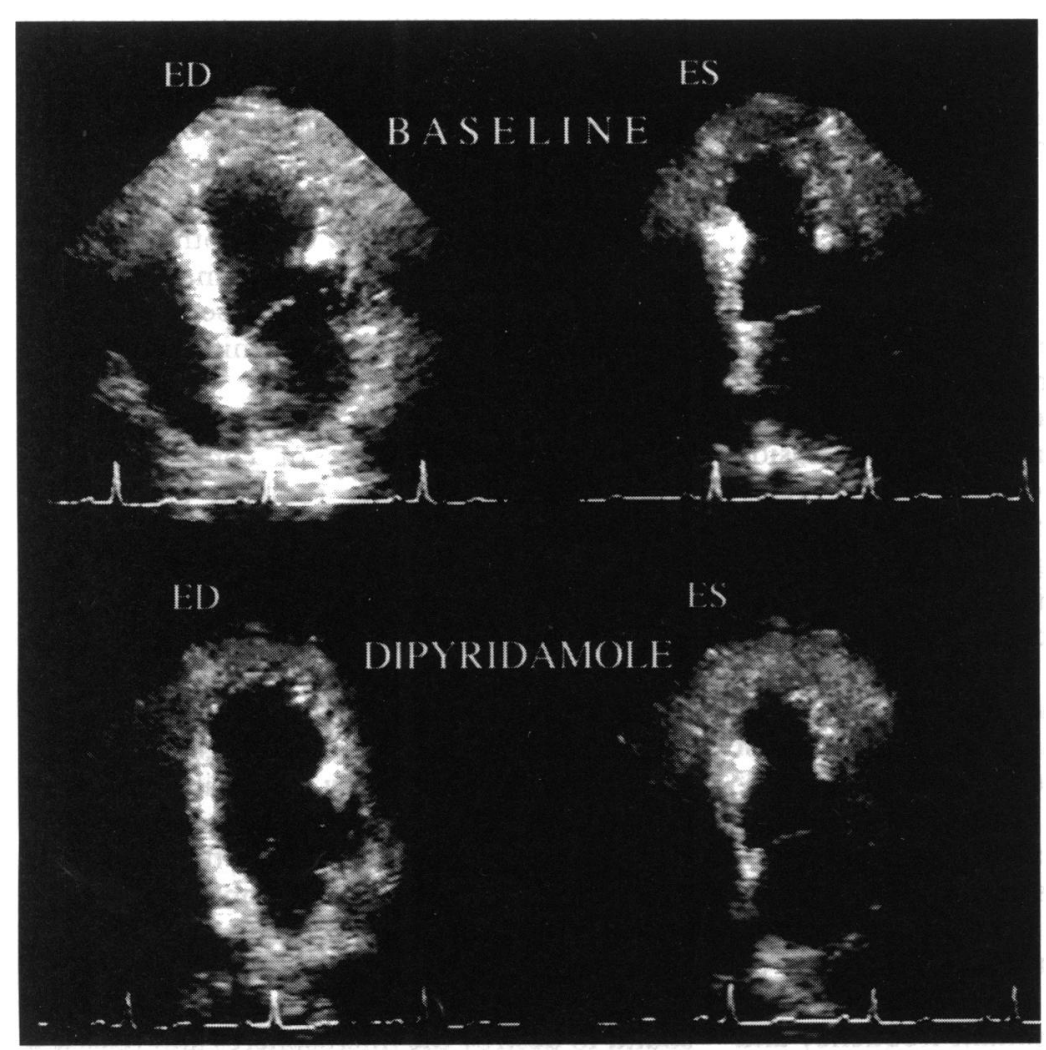

Figure 6 Contrast echo images obtained by the injection of Albunex into the left coronary artery of a patient, both at baseline and after low dose dipyridamole infusion. that allows analysis of the changes in 2nd harmonic (or other frequency characteristic) amplitudes before, during, and after contrast administration. Software that performs digital subtraction and colour coding on harmonic images is alse being generated and this should create colour coded perfusion images, similar to those obtained by nuclear medicine studies. Pseudo-colour maps are often used to enhance the detection of spontaneous echo contrast in the left atrial cavity or appendage and such specific display modalities might favour the detection of low concentrations of contrast agent. Although no studies have so far compared grey scale with colour images in the detection of myocardial perfusion abnormalities, it is expected that colour coded images could allow a better detection of myocardial contrast enhancement.

A further advancement might be the use of so called functional images, ${ }^{18}$ where the colour represents the value of a functional parameter (fig 5). Each of these two images derives from the analysis of a series of frames and the colours represent the time from contrast injection into the aortic root up to myocardial contrast appearance. Under baseline conditions, the myocardium appears homogeneously red, while it turns toward yellow in the area perfused by a stenotic left circumflex coronary artery because of a delay in myocardial contrast appearance. Although these imaging modalities may favour the detection and localisation of myocardial perfusion abnormalities, it is not clear what the best parameter will be to study myocardial perfusion by intravenous contrast echocardiography.

\section{Clinical models}

Experimental studies have shown that myocardial contrast echocardiography can differentiate normal from severely hypoperfused myocardium; however, the absolute measurement of coronary blood flow by this technique is still limited by several factors.

It is foreseeable that the categories of patients who might benefit more from new echocardiographic contrast agents and technologies are those in whom a semiquantitative assessment of myocardial perfusion is sufficient for clinical decision making. These patients should ideally present a normal perfusion pattern in certain areas of the myocardium and a severe hypoperfusion in other areas. Examples are with either acute or chronic myocardial infarction.

In patients with acute infarction, the end points of contrast echocardiography should be to detect myocardial perfusion abnormalities, to quantify the spatial extent of these abnormalities, to establish the occurrence of myocardial reperfusion, and thus to predict the late recovery of left ventricular function.

In patients with previous infarction, contrast echocardiography should provide indices of myocardial viability, based on the hypothesis that myocardial echo contrast effect reflects microvascular integrity and thus viability. ${ }^{19}$ Finally, contrast echocardiography should be 
compared with stress echocardiography with the end point of detecting myocardial ischaemia or viability. ${ }^{20}$ An example of combined stress and contrast echocardiography is shown in fig 6, where Albunex was injected into the left coronary artery of a patient with previous infarction both at baseline and after intravenous dipyridamole infusion.

\section{Conclusion}

After the initial observation that it was possible to study myocardial perfusion by echocardiography, following injection of contrast agents into the coronary circulation, different investigators, pharmaceutical, and instrument companies have contributed towards a progressive advancement of knowledge in this field. Once the new generation of echo contrast agents and scanners are available, an acceleration in this process can be expected. The combination of these efforts will provide a non-invasive, non-nuclear assessment of myocardial perfusion, preserving the high spatial and temporal resolution of ultrasound, in addition, being available in real time in a number of different clinical arena.

1 De Maria AN, Bommer WJ, Riggs K. Echocardiographic visualisation of myocardial perfusion by left heart and intracoronary injections of echo contrast agents [abstract]. Circulation 1980;62(Suppl III):III143.

2 Monaghan MJ, Quigley PJ, Metcalfe JM, Thomas SD, Jewitt DE. Digital subtraction contrast echocardiography: a new method for the evaluation of regional myocardial perfusion. Br Heart $\mathcal{F}$ 1988;59:12-19.

3 Feinstein SB, Cheirif J, Ten Cate FJ, Silverman PR, Heidenreich PA, Dick C, et al. Safety and efficacy of a new transpulmonary contrast agent: initial multicenter clinical results. $\Im$ Am Coll Cardiol 1990;16:316-24.

4 Schlief R, Staks T, Mahler M, Rufer M, Fritzsch T, Seifert W. Successful opacification of the left heart chambers W. Successful opacification of the left heart chambers injection of a new saccharide based contrast agent. injection of a new saccharide

5 Villaneuva FS, Glasheen WP, Sklenar J, Jayaweera AR Kaul S. Successful and reproducible myocardial opacification during two-dimensional echocardiography from right atrial injection of contrast. Circulation 1992;85. $1557-64$.

6 Grayburn PA, Erickson JM, Escobar J, Womack L, Velasco CE. Peripheral intravenous myocardial contrast echocar diography using a $2 \%$ dodecafluoropentane emulsion: identification of myocardial risk area and infarct size in the canine model of ischemia. $\mathcal{F}$ Am Coll Cardiol 1995; 26:1340-7.

7 Main ML, Escobar JF, Hall SA, Grayburn PA. Safety and efficacy of QW7437, a new fluorocarbon-based echocardiographic contrast agent [abstract]. F Am Coll Cardiol 1997;29(Suppl 2):299A.

8 Porter TR, Xie F. Transient myocardial contrast after initial exposure to diagnostic ultrasound pressures with minute doses of intravenously injected microbubbles. Demonstration and potential mechanisms. Circulation Demonstration

9 Dittrich HC, Bales GL, Kuvelas T, Hunt RM, McFerran BA, Greener Y. Myocardial contrast echocardiography in experimental coronary artery occlusion with a new intravenously administered contrast agent. $\mathcal{F} \mathrm{Am}$ Soc Echocardiogr 1995;8:465-74.

10 Schneider M, Arditi M, Barrau MB, Brochot J, Broillet A, Ventrone R, et al. BR1: a new ultrasonographic contrast agent based on sulfur hexafluoride-filled microbubbles. Investigative Radiology 1995;8:451-7.

11 Mulvagh SL, Foley DA, Aeschbacher BC, Klarich KK, Seward JB. A new intravenous perfluorochemical echocardiographic contrast agent, Imagent US: imaging characteristics and hemodynamic profile [abstract]. $\mathcal{F} \mathrm{Am} \mathrm{Soc}$ Echocardiogr 1995;8:345.

12 Sutherland GR, Grauer SE, Moran C, Ishii M, Sahn D. Aerosomes MRX 115 echo contrast agent demonstrates myocardial opacification after intravenous injection in mumans, without ignificant side effects, in a phection in ical trial. Circulation 1995;92(Suppl):2213.

13 de Jong N, Hoff L, Skotland T, Bom N. Absorption and scatter of encapsulated gas filled microspheres: theoretiscatter of encapsulated gas filled microspheres: theoreti1992;2:95-103.

14 Monaghan MJ, Metcalfe JM, Odunlami S, Thomas SD, Waaler A, Jewitt DE. Digital radiofrequency echocardiography in the detection of myocardial contrast following intravenous administration of Albunex. Eur Heart $\mathcal{F}$ 1993;14:1200-19.

15 Mulvagh SL, Foley DA, Aeschbacher BC, Klarich KK, Seward JB. Second harmonic imaging of an intravenously administered echocardiographic contrast agent: visualisation of coronary arteries and measurement of coronary blood flow. F Am Coll Cardiol 1996;27:1519-25.

16 Hancock J, Dittrich H, Jewitt DE, Monaghan MJ. Diagnostic ultrasound intensities may destroy contrast microspheres in-vivo: implications for echocardiographic myocardial perfusion imaging [abstract]. Heart 1996; 75(Suppl 1):P20.

17 Hancock J, Dittrich H, Jewitt DE, Monaghan MJ. New technologies for echocardiographic myocardial perfusion imaging [abstract]. Heart 1996;75(Suppl 1):P68.

18 Rovai D, Lombardi M, Ferdeghini EM, Marzilli $M$, Distante A, Benassi A, et al. Color-coded functional Distante A, Benassi A, et al. Color-coded functional
imaging of myocardial perfusion by contrast echocardiogimaging of myocardial perfusion by contrast ech

19 Ragosta M, Camarano G, Kaul S, Powers ER, Sarembock IJ, Gimple LW. Microvascular integrity indicates myocellular viability in patients with recent myocardial infarction. New insights using myocardial contrast echocardiography. Circulation 1994;89:2562-9.

20 Rovai D, Zanchi M, Lombardi M, Magagnini E, Chella P, Pieroni A, et al. Residual myocardial perfusion in reversibly damaged myocardium by dipyridamole contrast echocardiography. Eur Hear f 1996;17:296-301. 